\title{
Análisis espacial del sistema defensivo de la costa granadina en época nazarí y su transformación tras la conquista castellana
}

Spatial analysis of Kingdom of Granada's coastal defensive system in Nasrid period and its transformation after the Castilian conquest

\author{
Jorge Rouco Collazo $^{\text {a, }}$ José $M^{\text {a }}$ Martín Civantos ${ }^{b}$ \\ MEMOLab Laboratorio de Arqueología Biocultural - Universidad de Granada, Granada, Spain \\ a jroucocollazo@gmail.com; b civantos@ugr.es
}

\begin{abstract}
The coast of the Kingdom of Granada was a border of importance in Nasrid times and it was also a zone to be protected after the Castilian conquest, mostly against piracy. To control the sea, the successive rulers would build a system of fortresses and watchtowers. The objective of this paper is to apply spatial analysis to the defense system of the southern coasts of Granada and Almeria from the fifteenth to seventeenth century, focusing in its evolution. To achieve this objective, we will use historical documentation and archaeological record as main data for the spatial analysis carried out by a GIS software, mainly visibility and distribution. With this approach we expect to add new insights to this topic and stablish a base for further research and comparison with other neighboring areas of the Kingdom of Granada.
\end{abstract}

Keywords: Visibility, GIS, coastal defense, Granada.

\section{Introducción}

Desde que los grupos humanos descubren la navegación, las grandes masas de agua dejan de ser una barrera para convertirse en un espacio de comunicación e intercambio de personas, mercancías e ideas. Pero también se transforman en un área de peligro, al abrir la posibilidad de ataques y saqueos marítimos.

En época medieval y moderna, el mar se convertirá en otra frontera que, al igual que la terrestre, será un espacio de poblamiento y control en mayor o menor grado por parte de los distintos poderes. En esta comunicación aportamos nuevos datos para la comprensión del dispositivo de vigilancia y defensa costera del sector central del reino de Granada (desde la ensenada de $\mathrm{La} \mathrm{He}$ rradura hasta el Cabo de Gata) en los siglos XVI, XVII y XVIII mediante análisis espaciales con
Sistema de Información Geográfica (SIG). Si bien las fortificaciones en esta zona han recibido bastante atención desde el punto de vista histórico, sobre todo las de la Edad Moderna (Barea, 1987; Gil, 1998, 2004), apenas se ha explotado su potencial arqueológico y espacial (GarcíaConsuegra, Rodríguez, 2016; García-Consuegra, 2017; Villanueva, García, 2012).

\section{Metodología}

La base fundamental de este trabajo son los análisis espaciales mediante SIG, mayoritariamente visuales, por entender que es el mejor método para evaluar un sistema de vigilancia como el costero. No obstante, debemos indicar que un control visual no es nunca directamente 


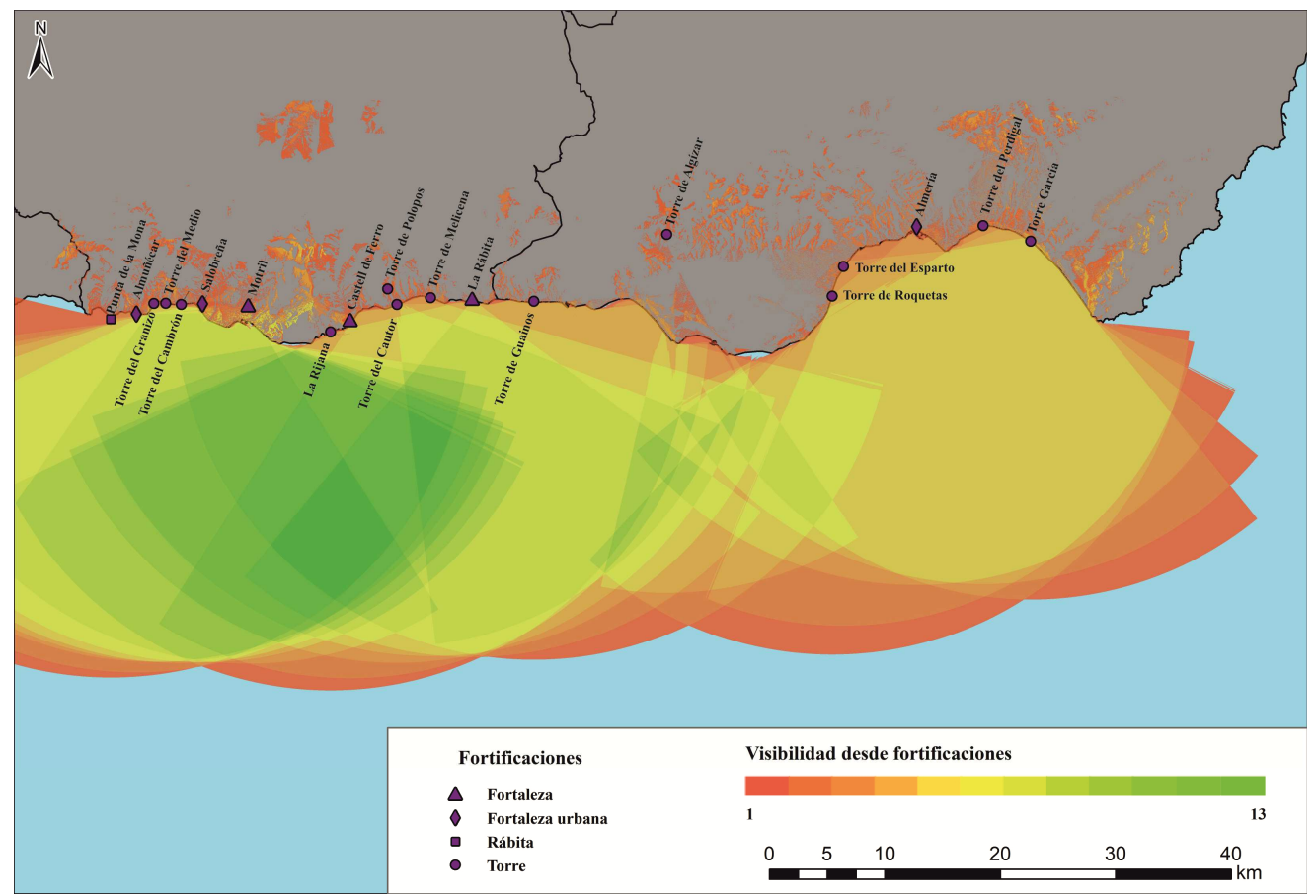

Fig. 1. Mapa de visibilidad acumulada de las fortificaciones costeras en el siglo XV (J. Rouco Collazo).

equiparable a un control efectivo, que requiere siempre un mayor número de efectivos en la zona.

El primer paso para cualquier estudio de este tipo es la obtención de unas planimetrías base que permitan ejecutar con éxito unos análisis de calidad. La cartografía base empleada es el Modelo Digital de Terreno del Instituto Geográfico Nacional, con una precisión de $5 \mathrm{~m}$, suficiente al estar trabajando con un área aproximada de $8.200 \mathrm{~km}^{2}$.

A continuación, se ha determinado el número de fortificaciones y torres ubicadas en la costa a partir de la historiografía para los siglos XV, XVI y XVII (Gil, 2004; Martín, Bleda y Martín, 1999; Sánchez, 1988). Se han establecido las fechas más probables de construcción y de abandono a partir de la documentación existente, sobre todo de las abundantes visitas de época castellana. La cronología de las torres de vigilancia en época medieval presenta más dificultades, puesto que al margen de las que han quedado fosilizadas en sus formas y las que podemos intuir como ya existentes por la Provisión de 1501, a menudo se ha tendido a atribuir fases medievales a estructuras de tipología moderna sin ninguna base material o documental que lo respaldase, por lo que se ha decidido no tenerlas en cuenta. Así, en total se han contabilizado un total de 20 fortificaciones para el siglo XV, 48 fortificaciones para el XVI ${ }^{1}$ y 47 para el XVII ${ }^{2}$. Estas se han ubicado según sus restos o, en el caso de haber desaparecido, se ha ubicado aproximadamente por la toponimia, la topográfia y las distancias con fortificaciones todavía existentes expresadas en las visitas de inspección.

Todos los análisis se han realizado con el programa ArcMap 10.2. A la hora de realizar el análisis de cuenca visual, otro de los factores principales, además del emplazamiento, es la altura del observador. En aquellas torres en las que se ha conservado el alzado hasta la azotea superior, se ha empleado esta medida más $1,7 \mathrm{~m}$ de la altura de un supuesto vigilante. En la mayoría en la que no se conserva el alzado original, se ha optado por establecer la altura aproximada en $11,7 \mathrm{~m}$, a partir de las dimensiones medias de los restos conservados. En el caso de las estancias, 
cuyos restos no han pervivido hasta día de hoy, se ha establecido una hipotética altura de observación de $5 \mathrm{~m}$, suponiendo la existencia de algún tipo de estructura de vigilancia de tamaño medio y al observador en su parte superior. La altura del punto observado se tiene en cuenta como 0 , a nivel del mar. De radio máximo de visión se ha utilizado $50 \mathrm{~km}$ como rango óptimo en condiciones de visibilidad atmosféricas favorables. Se tiene en cuenta, además, la curvatura de la tierra en el cálculo. Complementariamente, se han realizado análisis de intervisibilidad entre las fortificaciones, de distancia entre ellas y de densidad de Kernel para localizar los puntos clave de la red de vigilancia.

\section{El sistema de vigilancia nazarí en el siglo XV}

La costa adquiere en etapa nazarí una renovada importancia como frontera que, al igual que la terrestre, debe ser organizada y controlada. Este sistema se basará en fortalezas en las principales ciudades costeras como Salobreña, Almuñécar y Almería, y otras fortificaciones como la de Castell de Ferro (Martín, 1984) y la Rijana, junto con torres costeras y, probablemente, rábitas.

Los puntos centrales de esta red serían las grandes fortificaciones urbanas, más abundantes en la costa granadina. Aunque muchas de ellas tengan ya precedentes califales, caso de Almuñécar y Salobreña (Gómez, 1995 y 2000), alcanzan su máximo desarrollo en época nazarí en paralelo al crecimiento urbanístico, convirtiéndose la alcazaba de Salobreña en un espacio palatino ligado con los sultanes (Navarro, Orihuela y Reyes 2018). En ellas habría permanentemente un alcaide, delegado del poder central, con su guarnición. La fortaleza de Castell de Ferro, la fase nazarí de la Rijana (Malpica y Gómez, 1991), y quizás la etapa final medieval de la Rábita, también serían un tipo de fortificación ligada básicamente al control de la costa y no tanto a la defensa de la población, al no contar con grandes recintos que posibiliten un refugio temporal como es el caso de las fortificaciones de la Alpujarra Alta. En el caso de Castell de Ferro conocemos también su vinculación directa con el poder y la obligación de los habitantes de la zona de colaborar con el Estado nazarí en su mantenimiento mediante materias primas y mano de obra (Pérez, 1984; Malpica, 1986).

Estas fortalezas serían complementadas con torres atalayas de mampostería de diversa tipología, tanto cilíndricas como cuadrangulares, con un origen diverso. Es probable que algunas de ellas fuesen en sus inicios erigidas por las comunidades locales para proteger calas y pesquerías, como sucede con la del Cautor o la de Melicena (Malpica, 1986). Fuese quién fuese su promotor, para el siglo XV servirían sin duda como puntos de observación dentro de la red de defensa.

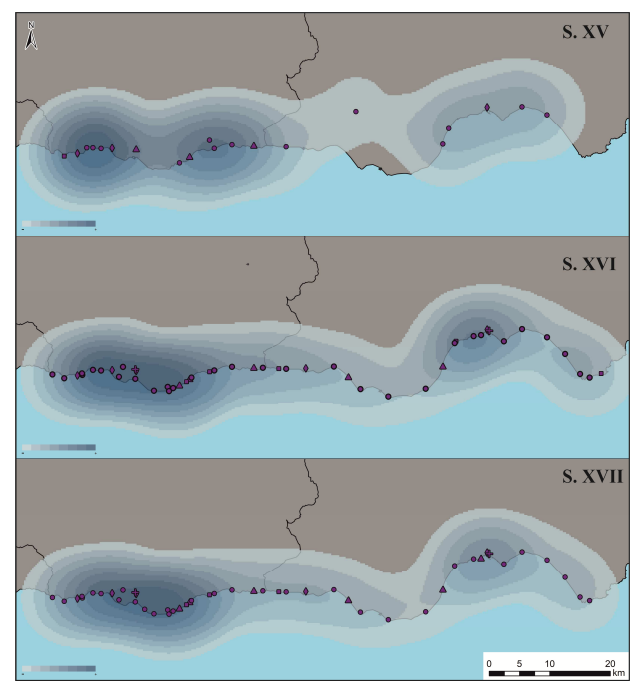

Fig. 2. Densidad Kernel de la red de fortificaciones (J. Rouco Collazo).

A través del análisis de cuencas visuales acumuladas y de densidad se puede observar cómo existen dos conjuntos diferenciados en la costa de Granada (Figs. 1 y 2). El primero de ellos sería el sector occidental, articulado en torno a Almuñécar y Salobreña, mientras que el segundo, en la parte oriental, estaría centrado por Castell de Ferro y la Rábita como principales puntos fuertes. Entre estos dos grupos, la costa del cabo Sacratif resultaría un punto ciego, pero las aguas al sur de este alcanzan el mayor cruce de visibilidad de todo el sistema, siendo visibles desde entre 10 y 13 puntos simultáneamente, por lo que podemos afirmar que aún en caso de malas 


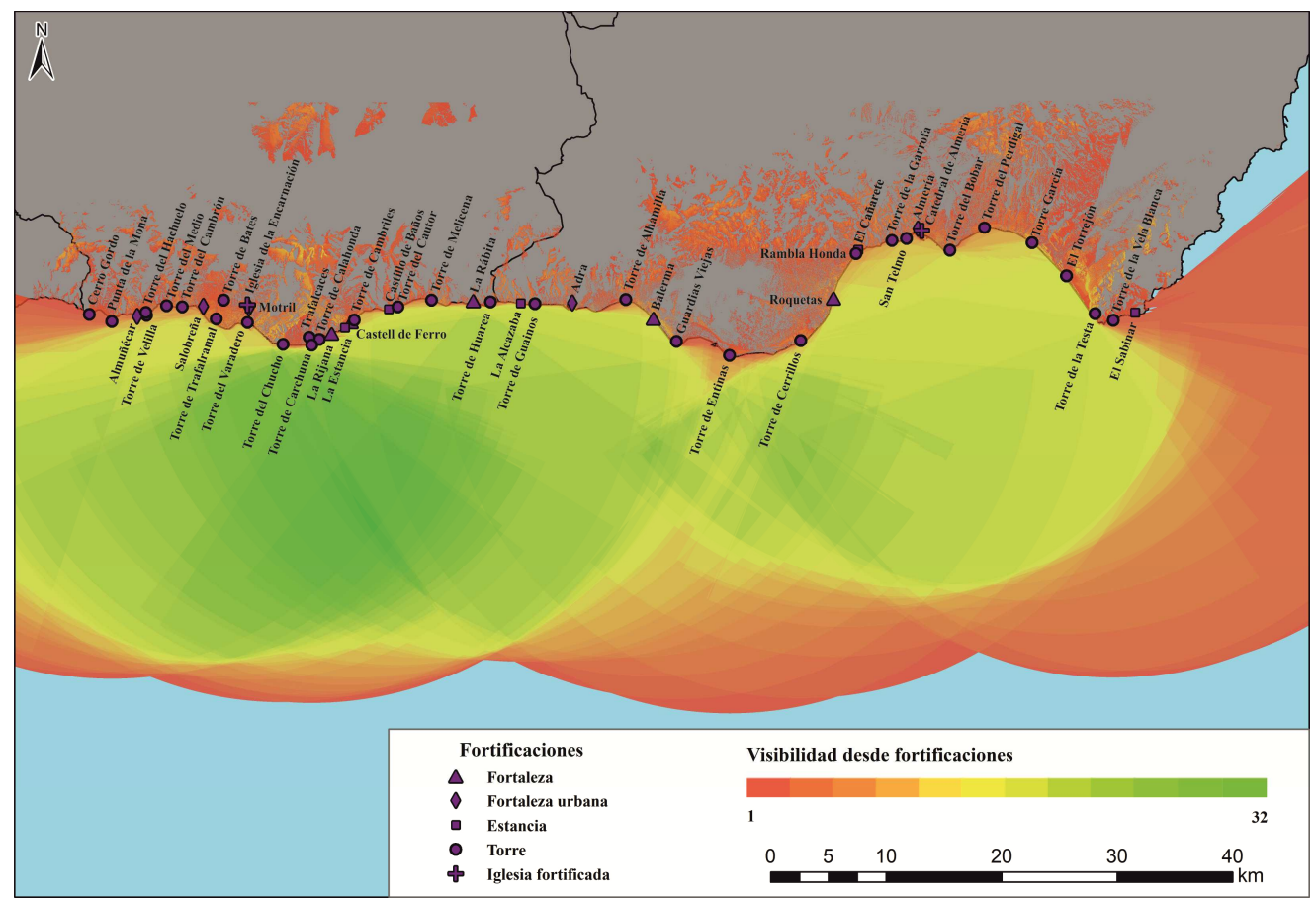

Fig. 3. Mapa de visibilidad acumulada de las fortificaciones costeras en el siglo XVI (J. Rouco Collazo).

condiciones de visibilidad, el sistema de control de la costa en la zona granadina sería bastante eficaz de estar convenientemente guarnecido. El nivel de intervisibilidad sería también muy alto entre los dos grupos y la mayoría de fortificaciones estarían a entre 1,5 y $3 \mathrm{~km}$ de distancia de la siguiente más cercana. Serían visibles además desde la mayor parte de alquerías y pesquerías costeras que están documentadas para este período (Malpica, 1989). No serían visibles sin embargo las alquerías ubicadas en las sierras costeras, cuya protección estaría basada en otro tipo de fortalezas, los husun.

Todo lo contrario podemos decir del sector almeriense. La alcazaba de Almería sería la única fortificación de entidad en esta parte de la costa. La bahía de Almería sí tendría una cobertura visual admisible, al cruzarse cinco puntos de observación. Sin embargo, el Campo de Dalías se encuentra en su mayor parte desprotegido, pues no hemos podido localizar con seguridad estructuras en este siglo más allá de la Torre de Roquetas y la del Esparto en la parte oriental del Campo (Cara y Cara, 1991). La torre de Algízar en la propia Dalías solo tendría visibilidad sobre una parte muy reducida de la costa. Este punto ciego se vería mitigado por la visibilidad desde las torres y fortalezas de la costa granadina, no obstante, estando la más cercana a $30 \mathrm{~km}$, en caso de que las condiciones climáticas no fuesen óptimas, un posible desembarco enemigo sería prácticamente indetectable. Esta inexistencia de fortificaciones en la parte meridional y occidental del Campo de Dalías podría deberse a la desaparición de sus vestigios materiales. Sin embargo, el hecho de que para la Provisión de 1501 (Gil, 2004) lo que se instale en este punto sean mayoritariamente estancias de vigilancia parece indicar que a finales del siglo XV no existirían torres en pie que pudiesen ser reutilizadas. La falta de defensas en el Campo de Dalías supuso un grave problema para sus habitantes, como registró el polígrafo lojeño Ibn alJatib: "los inconvenientes de este pueblo (Dalías) consisten en los muchos daños que le ocasionaban con sus frecuentes desembarcos las naves enemigas (...), era una senda de cuidados y martirios y sólo le frecuentaban varones de gran abnegación y desprecio por el mundo" (citado en 
Cara y Cara, 1991). Este será uno de los factores por los que se aprecia una ruptura en el poblamiento medieval de esta zona a partir del siglo XIII, desapareciendo el hábitat permanente (Cara y Cara, 1991).

\section{El sistema defensivo castellano en el siglo XVI}

Tras la conquista del reino granadino, el nuevo poder dispondrá de manera inmediata la reestructuración de la defensa costera. La monarquía castellana realizará un gran esfuerzo a lo largo del siglo XVI para tener un sistema de vigilancia y defensa de la costa, la nueva frontera una vez conquistado el reino de Granada, que impidiese por un lado los ataques vía marítima de corsarios y, por otro, la comunicación de la población morisca con el exterior.

Para ello, se establecerá un sistema de estancias y torres que otearían la costa, con torreros sufragados por la Corona, y de guarniciones que deberían acudir en caso de ataque. Inicialmente, este dispositivo sería financiado por la población recién conquistada mediante el sistema de far- das, impuesto que mantenía estas fortificaciones a cambio del permiso de seguir viviendo junto a la costa (López de Coca, 1976).

El nuevo sistema reutilizará en gran medida las estructuras ya presentes en la costa y edificará nuevas a lo largo del XVI, sustituyéndose paulatinamente las estancias por atalayas para garantizar una visión de toda la costa y la intervisibilidad entre los distintos puntos, estando estipulado el mecanismo de señales mediante hogueras. Las torres erigidas durante este siglo son de planta circular, de forma ligeramente troncocónica, realizadas en mampostería y con el acceso por escala a $4 \mathrm{~m}$ de altura. Sus fechas de construcción y su estado son mucho mejor conocidos que en el caso de las fortificaciones del siglo XV por haber conservado la documentación de un gran número de visitas de inspección e informes de obras y reparaciones al ser dependientes directamente de la Corona.

Para finales del XVI, el número de puntos de observación habrá aumentado notablemente de 20 a 48, con la correspondiente mejora del control visual de la costa. De esta forma, los principales

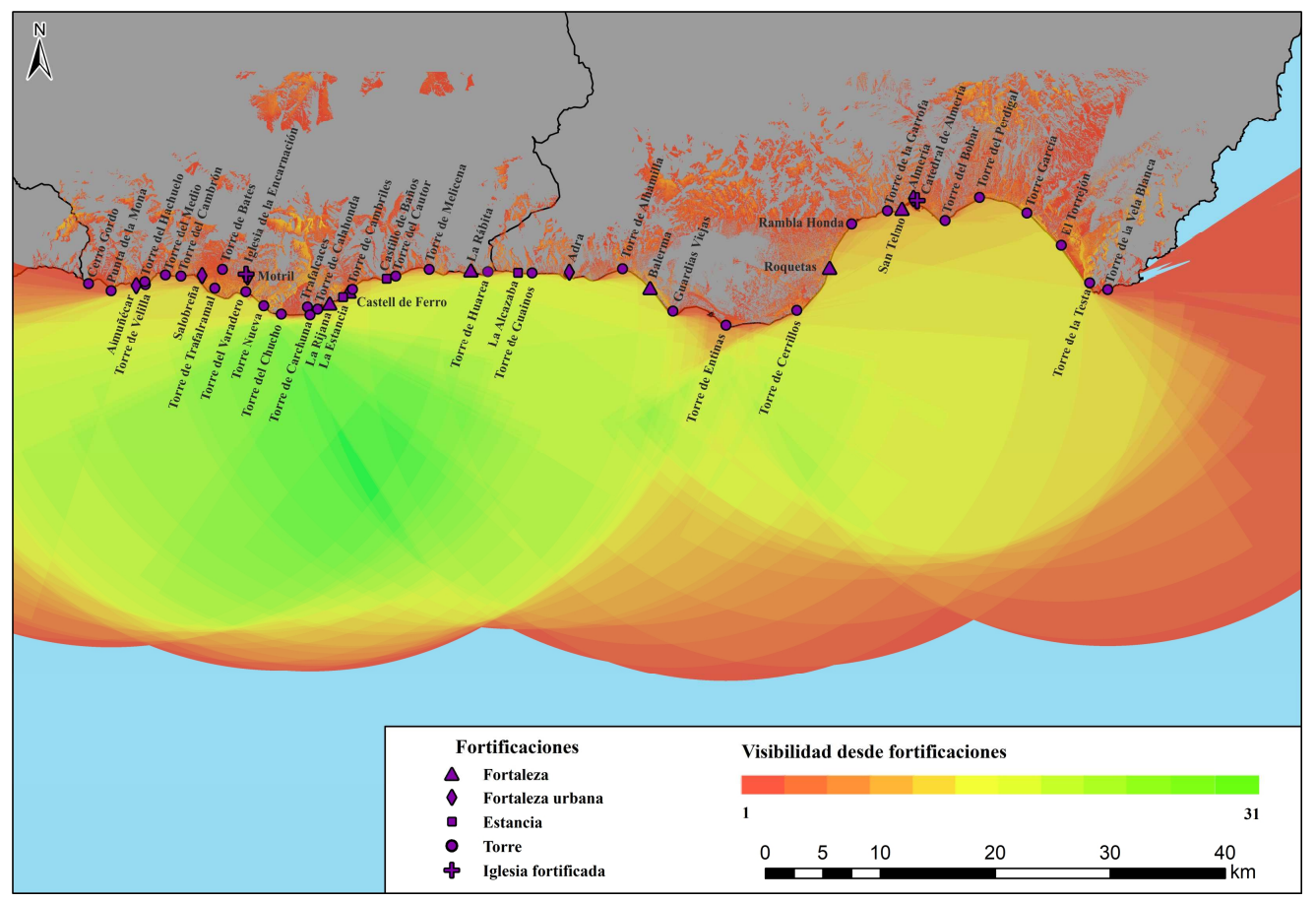

Fig. 4. Mapa de visibilidad acumulada de las fortificaciones costeras en el siglo XVII (J. Rouco Collazo). 
puntos ciegos del sistema en el siglo XV serían eliminados. Para ello, en el cabo Sacratif, en Granada, se erigen las torres del Chucho, de Carchuna y Calahonda, mientras que en el Campo de Dalías se corrige paulatinamente su clara indefensión (Gil, 2004). Se pasa de las varias estancias citadas en el Ordenamiento de 1501 a la existencia de las torres de Alhamilla, Guardias Viejas, Entinas y Cerrillos y la fortaleza de Balerma en la parte sur y occidental. Se traslada además la localidad de Adra junto a la costa y la torre de Roquetas se transforma en una fortaleza, habiendo una fortificación de importancia en cada sector del Campo de Dalías. En la bahía de Almería también se incrementará notablemente el número de fortificaciones, incluyendo el Cabo de Gata (Fig. 3).

No obstante, la costa granadina continuará siendo la zona con mayor visibilidad acumulada, llegando a cruzarse hasta 32 puntos de visión. El análisis de densidad confirma todavía el mayor peso de la zona granadina, habiéndose unificado en una única gran zona de influencia frente a las concentraciones más dispersas de la parte almeriense. La distancia entre fortificaciones se reducirá también considerablemente de media, estando la mayoría a menos de $2 \mathrm{~km}$ de la más próxima, favoreciendo esto la intervisibilidad de las mismas. Esta mejora de la infraestructura, sin embargo, debe tomarse con cautela, ya que en las visitas de inspección no resulta extraño que el visitador encuentre que la torre se encuentra desatendida, aun en los casos en los que la estructura estuviese en óptimas condiciones.

\section{El sistema defensivo en el siglo XVII}

El siglo XVII supone una neta continuación del sistema del XVI, estando en funcionamiento prácticamente el mismo número de fortificaciones y torres (Fig. 4). La corona de los Austrias, con bastantes problemas financieros en este siglo, se dedicaría básicamente al mantenimiento, mejora y sustitución de las torres más dañadas. La única torre de nueva planta que parece haber sido construida en este siglo sería la de Torrenueva, en Motril (García-Consuegra, Rodríguez, 2016). Se produce en estos momentos también cierta privatización de la vigilancia, cediéndose a particulares la tenencia de diversas fortificaciones y torres tras el terremoto de1658 a cambio de su reparación (Gil, 2004, pp. 43-45).

Los análisis visuales serán por tanto prácticamente idénticos, manteniéndose el sistema sin novedades hasta los cambios introducidos en el siglo XVIII, con la construcción de baterías de costa y el Reglamento de 1764 (Gil, 2004, pp. 1151-1202), momento que va más allá del arco temporal de este estudio.

\section{Conclusiones}

Los distintos poderes que se suceden en el reino de Granada tendrán un interés manifiesto en la defensa de la costa y de sus recursos, como parte de la frontera. Las alcazabas de las medinas costeras, junto con las torres atalaya, articularían un sistema de vigilancia enfocado mayoritariamente en el control y la defensa de las zonas más pobladas y explotadas económicamente de la costa, como muestra la mayor acumulación de fortificaciones en la parte granadina, frente a la costa almeriense. Estas estarían relacionadas directamente con el sultán por delegación en el alcaide y su guarnición, pero la población estaría obligada a colaborar en su mantenimiento mediante la aportación de materias primas y trabajo. Este sistema ligado al Estado sería bastante diferente a las fortificaciones de defensa comunitaria situadas más al norte, en la Alpujarra Alta, cuyo objetivo principal sería guarecer a la población y no crear una red de vigilancia, vinculadas más por tanto a la iniciativa campesina.

Tras la conquista castellana, las fortificaciones costeras nazaríes serán el punto de partida para la base del nuevo sistema defensivo costero de los siglos XVI y XVII, en el que intervendrá directamente la Corona. Las viejas fortificaciones serán reparadas y otras construidas de nueva planta en los puntos más desprotegidos de la costa, principalmente el Campo de Dalías. La aglomeración defensiva principal continúa siendo la costa granadina. Esto se debe en parte al peso previo del sistema nazarí y seguramente también a la mayor concentración de población en este sector, además de la orografía montañosa de esta costa, que obliga a un mayor número de 
puntos de observación para el control de las numerosas calas y fondeaderos.

El análisis espacial mediante SIG ha resultado útil para conocer en profundidad el diseño de la vigilancia costera y hasta qué punto este respondería a un plan centralizado. Sería interesante en el futuro realizar otro tipo de análisis, como los de visibilidad difusa, en la línea del realizado dentro de los estudios de paisaje marítimo (Cerezo-Andreo, Pérez-Reverte, Maria, 2016; Cerezo-Andreo, 2017), para precisar a partir de qué punto puede distinguirse desde la costa una embarcación acercándose y tener en cuenta las condiciones climáticas habituales en la zona. También lo sería ampliar el trabajo a la costa malagueña y al Levante almeriense, para poder obtener una visión de conjunto de la evolución de la vigilancia costera en todo el reino nazarí y su evolución en época moderna, en conjunción con los datos arqueológicos e históricos disponibles.

\section{Notas}

${ }^{1}$ Incluyendo para el siglo XVI y XVII las iglesias-fortaleza de la Catedral de Almería y Nuestra Señora de la Encarnación de Motril por ubicarse en villas costeras de importancia. Para mayor conocimiento de las iglesias fortificadas de la costa de Granada, véase la obra de M. Martín (2012, 2013).

${ }^{2}$ Se recomienda consultar el material adicional para más información y para la visualización de las imágenes en su resolución original en https://digibug.ugr.es/handle/10481/58382.

\section{Bibliography}

Barea, J.L. (1987). La defensa de la costa del Reino de Granada en la época de los Austrias, Granada Ed., Granada.

Cara, L.; Cara, J. (1991). "El poblamiento andalusí en el campo de Dalías oriental (Almería): discontinuidades y permanencias", Boletín del Instituto de Estudios Almerienses, 11-12, pp. 21-58.

Cerezo-Andreo, F. (2017). "Los puertos antiguos de Carthago Nova, nuevos datos desde la arqueología marítima y geoarqueología porturaria”, in Los puertos atlánticos béticos y lusitanos y su relación comercial con el Mediterráneo, Universidad de Huelva Ed., Huelva, pp. 436-474

Cerezo-Andreo, F.; Pérez-Reverte, C.; Maria, C. (2016). "New approaches to maritime visibility: a comparative study of traditional mathematical methods, GIS application and experimental archaeology", in Actas del V Congreso Internacional de Arqueología Subacuática, Ministerio de Educación, Cultura y Deporte Ed., Madrid. pp. 288-290

García-Consuegra, J.M. (2017). "Un museo al aire libre desaprovechado. El sistema defensivo de la Costa Tropical granadina en Época Moderna. Una visión global de las estrategias defensivas contra piratas y corsarios", Bastetania, 5, pp. 61-85.

García-Consuegra, J.M.; Rodríguez, Á. (2016). “Arqueología de la defensa de la costa del Reino de Granada en época moderna. La torre vigía de Torrenueva (Motril, Granada)", Revista del Centro de Estudios Históricos del Reino de Granada, 28, pp. 231-267.

Gil, A. (1998). "Las fortificaciones de la costa del Reino de Granada (España). Estado de la cuestión”. Biblio 3W. Revista Bibliográfica de Geografía y Ciencias Sociales, 72.

Gil, A. (2004). Documentos sobre la defensa de la costa del Reino de Granada (1497-1857), G.B.G. Ed., Barcelona.

Gómez, A. (1995). "El poblamiento altomedieval en la costa de Granada”, Studia Historica, Historia medieval, 13, pp. 59-92.

Gómez, A. (2000). "El litoral granadino en época altomedieval (siglos VII-XI). Poblamiento, navegación y defensa", Arqueología y Territorio Medieval, 7, pp. 7-21.

López de Coca, J.E. (1976). "Financiación mudéjar del sistema de la vigilancia costera en el reino de Granada (14921501)", Historia, Instituciones, Documentos, 3, pp. 399-415. 
Malpica, A. (1986). "Castillos y sistemas defensivos en las ta’as alpujarreñas de Sahil y Suhayl: un análisis histórico y arqueológico", in Actas del I Congreso de Arqueología Medieval Española, Diputación General de Aragón Ed., Zaragoza, vol. 3, pp. 357-371.

Malpica, A. (1989). "Estructura de poblamiento de la Costa de Granada a fines de la Edad Media", Studia Historica, Historia medieval, 7, pp. 157-186.

Malpica, A.; Gómez, A. (1991). Una cala que llaman La Rijana. Arqueología y paisaje, Ayuntamiento de Gualchos Ed., Castell de Ferro.

Martín, M. (1984). Castell de Ferro, su castillo y torres almenaras: datos para su historia, Colegio Oficial de Aparejadores y Arquitectos Técnicos Ed., Granada.

Martín, M. (2012). "Iglesias fortificadas en la costa granadina”. in IV Congreso Nacional de Castellología, Asociación Española de Amigos de los Castillos Ed., Madrid, pp. 713-734

Martín, M. (2013). "Iglesias fortificadas del Reino de Granada", in Actas del Octavo Congreso Nacional de Historia de la Construcción, Instituto Juan de Herrera Ed., Madrid.

Martín, M.; Bleda, J.; Martín, J.Mª (1999). Inventario de arquitectura militar en la provincia de Granada, Diputación Provincial de Granada Ed., Granada.

Navarro, J.; Orihuela, A.; Reyes, A. (2018). "Salobreña. De alcazaba palatina nazarí a fortificación castellana en la costa de Granada", in Alborán. Poblamiento e intercambios en las zonas costeras de al-Andalus y el Magreb, Alhulia Ed., Granada, pp. 111-197.

Pérez, J. (1984). "El poblamiento de la Taha de Suhayl a fines de la Edad Media: el castillo de Castell de Ferro", Cuadernos de Estudios Medievales y Ciencias y Técnicas Historiográficas, 12-13, pp. 139-153.

Sánchez Sendano, Mª del P. (1988). Arquitectura musulmana en la provincia de Almería, Instituto de Estudios Almerienses Ed., Almería.

Villanueva, E.; García, A.M. (2012). "Comunicación visual mediante técnicas GIS en el litoral del Campo de Dalías: la defensa costera de los pueblos del interior", Virtual Archaeology Review, 3, pp. 93-97. 